\title{
Age-related changes in European Portuguese vowel acoustics
}

\author{
Luciana Albuquerque $e^{1,2}$, Catarina Oliveira ${ }^{1,3}$, António Teixeira $^{1,4}$, Pedro Sa-Couto ${ }^{5}$, Daniela \\ Figueiredo ${ }^{2,3}$ \\ ${ }^{1}$ IEETA, University of Aveiro, Portugal \\ ${ }^{2}$ CINTESIS.UA, University of Aveiro, Portugal \\ ${ }^{3}$ School of Health Science (ESSUA), University of Aveiro, Portugal \\ ${ }^{4}$ Dep. Electronics, Telecommunications and Informatics (DETI), University of Aveiro, Portugal \\ ${ }^{5}$ CIDMA/ Department of Mathematics (DMAT), University of Aveiro, Portugal

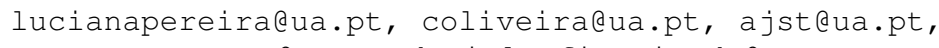

\begin{abstract}
This study addresses effects of age and gender on acoustics of European Portuguese oral vowels, given to the fact of conflicting findings reported in prior research. Fundamental frequency (F0), formant frequencies (F1 and F2) and duration of vowels produced by a group of 113 adults, aged between 35 and 97 years old, were measured. Vowel space area (VSA) according to gender and age was also analysed. The results revealed that the most consistent age-related effect was an increase in vowel duration in both genders. F0 decreases above [50-64] for female and for male data suggests a slight drop over the age range [3564] and then an increase in an older age. That is, F0 tends to be closer between genders as age increases. In general, there is no evidence that $\mathrm{F} 1$ and $\mathrm{F} 2$ frequencies were lowering as age increased. Furthermore, there were no changes to VSA with ageing. These results provide a base of information to establish vowel acoustics normal patterns of ageing among Portuguese adults.
\end{abstract}

Index Terms: ageing, acoustics, vowels, European Portuguese.

\section{Introduction}

From young adulthood to old age, the speech production mechanism undergoes several anatomical and physiological changes. Moreover there are substantial gender differences in the extent and timing of the ageing process $[1,2,3]$. Numerous studies have evaluated the effects of ageing on the acoustic properties of speech (e.g. [1, 3, 4]). Most of them have focused on fundamental frequency (F0) and have shown a decrease in F0 with ageing in women $[5,6,7,3,8,9]$; for men there is less agreement across researches, with some studies indicating that F0 significantly decreases above $60[4,10]$, and others suggesting an F0 drop in men over the age range 30-50 and then an increase in F0 in older age $[1,7,2,3,11,8,12,5]$.

Other studies have reported on age-related changes to formants (mostly F1 and F2), particularly in the production of vowels. The results are quite variable and the most consistent effect is an age-dependent formant frequency lowering [1, 13, 14]. Another finding is of a greater centralization of the vowel space in older speakers (which should result in movement to the centroid of formant space) $[15,16,3,8]$. In some cases the changes have been identified as occurring only on particular vowels and some studies have showed a gender-vowel interaction in formant frequencies $[3,5,17]$.

It has often been noted that older adults use slower speaking rates $[1,3,18]$. That is, vocal ageing implies a decrease in the number of syllables and phonemes per second, which leads to the increase of segment duration [19, 1, 3, 17].

Unlike other languages, only a few studies have been concerned with effects of ageing on F0 [20, 21, 22], duration and formant frequencies $[21,22]$ of European Portuguese (EP) vowels. Given that those previous researches used different methods and analysis procedures, it is hard at this time to draw solid conclusions on the effects of age and gender on EP vowel acoustics. Our previous study [22], with speakers between 60 and 90 indicated a slight age-related F0 decrease in women and a trend of increase in men. Vowel duration has shown to significantly increase with ageing. Age differences in vowel formant frequency were also observed, mainly in women. The comparison between our data and the results of previous studies on acoustic correlates of EP vowels from young adults [23] suggested a trend towards the centralization of the vowel space.

The purpose of this article is to analyse the effects of age and gender on duration, F0 and formant frequencies (F1, F2) for EP oral vowels. This study extends previous research by reporting data from four adult age groups, covering the age range of 35 to 97, which is essential to provide a more complete view of agerelated changes in EP vowel acoustics. The speech stimuli were carefully chosen to allow easy and accurate formant measure, and the greater constancy of the speech stimuli across speakers throughout the life span also facilitates comparisons and reduces variability. Since there is a paucity of literature on EP vowel acoustics $[23,24,22,25]$, this study also provides valuable insights to an accurate description of these sounds.

This study also examines the relationship between age, gender and Vowel Space Area (VSA). VSA is used to model possible reduction in the articulatory capability of speakers. Such reduction is observed as a compression of the area of the vocal space. The main hypothesis is that young speakers have a better articulation capability than older speakers $[26,27]$.

\section{Method}

\subsection{Participants}

113 native Portuguese speakers (56 men and 57 women), from the center region of Portugal, aged between 35 and 97, participated in this cross-sectional study. They were divided into 4 age groups: [35-49] (15 men, 15 women), [50-64] (15 men, 15 women), [6579] ( 15 men, 16 women), and $\geq 80$ ( 11 men, 11 women). They all completed a background questionnaire and signed a written informed consent form. All participants reported no previous history of speech-language impairments, head/neck cancer and/or 
neurological disorders. They were free of upper respiratory tract infection, and were excluded: 1) if they were current smokers or had smoked within the previous 5 years; 2 ) if they reported poor general health; and 3) if they wore hearing aids.

\subsection{Speech sample}

The corpus consisted of 36 words, with the vowels of the EP $[\mathrm{i}],[\mathrm{e}],[\varepsilon],[\mathrm{a}],[\mathrm{o}],[\mathrm{o}],[\mathrm{u}]$ in stressed position and the vowels $[\mathrm{i}]$ and $[\mathrm{e}]$ in unstressed position. Each vowel was produced in a disyllabic sequence, mostly CV.CV (C-consonant, V-vowel) (e.g. "pato", "duck"), where $\mathrm{C}$ was a voiced/ voiceless stop consonant $([\mathrm{p}],[\mathrm{t}],[\mathrm{k}],[\mathrm{b}],[\mathrm{d}],[\mathrm{g}])$ or a voiced/ voiceless fricative consonant ([f], [s], [ []], [v], [z], [3]). The stimuli were embedded in a carrier sentence "Diga ... por favor" ("Say ... please"). For each vowel, four different words were selected.

\subsection{Recording Protocol}

Recordings took place in quiet rooms, using an AKG C535 EB cardioid condenser microphone connected to an external 16-bit sound system (PreSonus Audio-BoxTM USB), at a sampling rate of $44100 \mathrm{~Hz}$. The sentences were randomized and presented individually on the computer screen with software system SpeechRecorder [28] using pictures together with the orthographic word. Participants read the sentences at comfortable pitch and loudness level, after familiarizing themselves with the sentences. Each carrier sentence was repeated 3 times. Thus, each participant produced 12 repetitions of each vowel, in a total of 108 productions by speaker (113 participants x 36 words x 3 repetitions $=12204$ recordings) .

\subsection{Segmentation of the Data Set}

The recorded data was first automatically segmented at phoneme level using WebMAUS [29] and then imported into Praat [30], so that 3 trained analyzers could manually check the accuracy of all phoneme boundaries, by finding the first and last positive zero crossings of the quasi-periodic waveform associated with the vowel. Recordings presenting clipping or other recording artifacts (e.g. noise, cough) or in which the speaker produced unusual hoarseness or vocal fry were excluded. In case the participants misread a word, it was not analysed. Furthermore, due to vowel reduction affecting unstressed vowels in EP, the vowel [i] was often elided [31]. A total of 758 recordings were not analysed (approximately $6.2 \%$ of trials).

\subsection{Acoustic measurements}

Acoustic parameters of the vowels were automatically extracted from the data set using Praat scripts. The F0 of the vowels was estimated with the cross-correlation algorithm, which is especially suitable for measuring short vowels [23]. Median F0 value was taken from the central $40 \%$ of each target vowel, which minimizes the impact of flanking consonants on the F0; in addition, taking the median F0 values rather than the mean, reduces the effect of F0 measurement errors [23]. The pitch range for the analysis was set to $60-400 \mathrm{~Hz}$ for men and $120-400 \mathrm{~Hz}$ for women. If the analysis failed on any of the speaker's vowel tokens, which only occurred for women, a new analysis was automatically performed using a pitch floor of $75 \mathrm{~Hz}$ (which occurred in only 28 of the 11446 vowel tokens, almost all from an 80 year old woman). Burg-LPC algorithm was used to compile values for $\mathrm{F} 1$ and $\mathrm{F} 2$, at the central $40 \%$ of the vowel. A procedure (adapted from [23] and previously used in [24, 22]), was applied to optimize the formant ceiling for a certain vowel of a certain speaker. The first two formants were determined 201 times for each vowel, for all ceilings between 4500 and $6500 \mathrm{~Hz}$ in steps of $10 \mathrm{~Hz}$ (for female), and for all ceilings between 4000 and $6000 \mathrm{~Hz}$ in steps of $10 \mathrm{~Hz}$ (for male). The chosen ceiling was the one that yielded the lowest variation (for more details see [23]). Thus, for each vowel produced by each speaker there is only one optimal ceiling. The duration measurements were computed from the label files with reference to the beginning and the ending points of each vowel. Vowels with duration values shorter than $20 \mathrm{~ms}$ were excluded (8 vowels). An important goal for this database is to provide normative data for adult age speakers, therefore, outliers that exceeded 2.5 standard deviations from the mean for particular speaker by $\mathrm{F} 0$ and from their gender $\mathrm{x}$ vowel mean by $\mathrm{F} 1$ and $\mathrm{F} 2$ were excluded from this analysis [5, 32] This procedure yielded in 532 outliers excluded from the study. The VSA is defined by the polygon area based on the mean value for each oral vowel, adapted from [26, 33, 34].

\subsection{Statistical Analysis}

The statistical analysis was conducted with the SPSS software package (SPSS 25.0 - SPSS Inc., Chicago, IL, USA). The values of F0, F1, F2 and duration were calculated for all productions, and subsequently, the median of repetitions was performed for each vowel and speaker. The VSA was calculated in $\mathrm{Hz}^{2}$ for each speaker. For each dependent variable (F0, F1, F2, and duration), a three-way mixed analysis of variance (ANOVA) was applied, with vowel as a within-subject factor and with gender and age as between-subject factors. For VSA, a two-way ANOVA was applied, with gender and age as between-subject factors. The ANOVA assumptions of residual normality and homogeneity of variance were validated (except homogeneity of variance for VSA). On what concerns the sphericity assumption, the Epsilon Huynh-Feldt correction was used. In all statistical analysis, the level of significance was $\mathrm{p}<0.05$.

\section{Results}

This section presents the detailed results of the acoustic measurements and statistical analysis aimed at investigating the effects of age, gender and vowel on duration, F0, formants, and VSA Table 1 summarizes the average values for all these parameters; each number is an average of the 9 oral vowels under analysis by gender and age group.

Table 1: Averages of vowel duration, FO, F1 and F2 by gender and age. ㅇ: women; $0^{x}:$ men.

\begin{tabular}{cc|c|c|c|c|c} 
& & Dur. & F0 & F1 & F2 & VSA \\
\hline \hline \multirow{9}{*}{} & $35-49$ & 104.8 & 197.0 & 489.8 & 1679.4 & 519944.3 \\
& $50-64$ & 112.3 & 204.8 & 498.8 & 1683.3 & 473407.7 \\
& $65-79$ & 126.9 & 185.8 & 477.5 & 1702.2 & 495234.4 \\
& $\geq 80$ & 125.3 & 175.4 & 476.1 & 1673.0 & 473500.2 \\
\hline \hline$\sigma^{7}$ & $35-49$ & 106.7 & 141.0 & 438.7 & 1441.5 & 334114.2 \\
& $50-64$ & 110.8 & 133.7 & 434.3 & 1429.3 & 312040.6 \\
& $65-79$ & 114.6 & 136.2 & 438.7 & 1433.5 & 313986.5 \\
& $\geq 80$ & 129.4 & 148.7 & 436.4 & 1426.3 & 269505.5
\end{tabular}

\subsection{Vowel Duration}

The results of EP vowel duration measurements by age and vowel are displayed in Figure 1. As can be seen, the duration of all vowels increased with ageing ([35-49] - $105.7 \mathrm{~ms} \pm 15.2$; [50- 
$64]-111.5 \mathrm{~ms} \pm 15.0 ;[65-79]-121.2 \mathrm{~ms} \pm 17.6$ ), and the older speakers presented the longest vowel duration (127.4 $\pm 24.4 \mathrm{~ms})$. ANOVA showed that the effect of age on vowel duration was statistically significant $(\mathrm{F}(3 ; 103)=7.4 ; \mathrm{p}<0.001)$. Statistical analysis also revealed that the duration was influenced by vowel $(\mathrm{F}(3.5 ; 361.4)=578,3 ; \mathrm{p}<0.001)$. The following pattern of vowel duration was observed: $[\mathrm{p}]<[\mathrm{i}]<[\mathrm{i}]<[\mathrm{u}]<[\varepsilon]<[\mathrm{o}]<[\mathrm{o}]<$ $[\mathrm{a}]<[\mathrm{e}]$. There were no significant differences $(\mathrm{F}(1 ; 103)=0.3$; $\mathrm{p}=0.572)$ in average vowel duration between male $(114.3 \mathrm{~ms})$ and female $(116.8 \mathrm{~ms})$ speakers. However, analysis indicated an interaction between vowel and age $(\mathrm{F}(10.5 ; 361.4)=3,2 ; \mathrm{p}<0.001)$. As can be seen in Figure 1, unlike the stressed vowels, the unstressed vowels ([i] and $[\mathrm{e}]$ ) did not show relevant changes of duration with age.

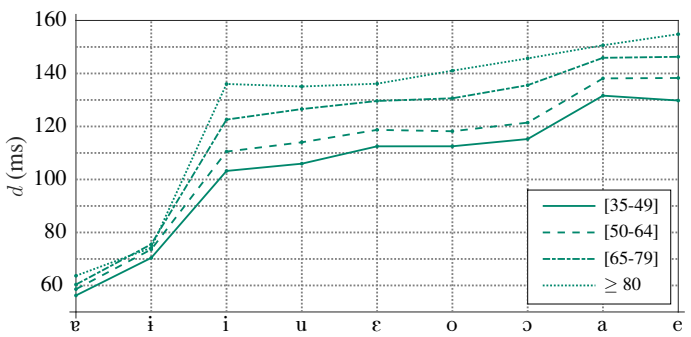

Figure 1: Mean vowel duration by age group.

\subsection{Fundamental Frequency}

Figure 2 shows that male speakers had significantly lower F0 $(139.3 \mathrm{~Hz} \pm 27.4)$ compared to female speakers $(192.0 \mathrm{~Hz}$ $\pm 25.2)$ regardless of age $(\mathrm{F}(1 ; 103)=106.5 ; \mathrm{p}<0.001)$. Findings also showed significant age by gender interaction for $\mathrm{F} 0$ $(\mathrm{F}(3 ; 103)=3.2 ; \mathrm{p}=0.028)$, indicating that the differences among age groups varied by gender. In male, F0 decreased until the age group [50-64] and started to increase after that age, with a more pronounced increase in the group $\geq 80$, that presented the highest mean value of F0. The opposite tendency was observed for female speakers, where F0 increased until the age group [5064] and started to decrease sharply after this age. The age group $\geq 80$ presented the highest mean value of F0. As illustrated in Figure 2, there were F0 differences between vowels of different phonological heights. Central low vowel [a] displays the lowest mean value of F0 followed by the mid vowels $([\varepsilon]<[0]<[\mathrm{i}]<$ $[\mathrm{e}]<[\mathrm{e}]<[\mathrm{o}])$ and finally, the high vowels $([\mathrm{i}]<[\mathrm{u}])$. The effect of vowel on $\mathrm{F} 0$ is statistically significant $(\mathrm{F}(1.8 ; 180.3)=33.8$; $\mathrm{p}<0.001)$. Additionally, there were detected significant interactions between vowel and gender $(\mathrm{F}(1.8 ; 180.3)=9,8 ; \mathrm{p}<0.001)$ and between vowel and age group $(\mathrm{F}(5.3 ; 180.3)=2,7 ; \mathrm{p}=0.019)$. As seen in Figure 2, for unstressed vowels F0 decreases very markedly with age.

\subsection{Formant Frequencies}

Figures 3 b) c) show the mean F1 and F2 values of the EP vowels for the four age groups, by gender. As expected, women presented higher F1 and F2 frequencies than men (cf. Table 1), regardless of age. The analysis of variance revealed a main effect of gender on both $\mathrm{F} 1(\mathrm{~F}(1 ; 103)=82.8 ; \mathrm{p}<0.001)$ and $\mathrm{F} 2$ $(\mathrm{F}(1 ; 101)=426.2 ; \mathrm{p}<0.001)$. As shown in Table $1, \mathrm{~F} 1$ and $\mathrm{F} 2$ values tended to be similar across the four age groups. As represented in Figure 3 a) and Figure 4 (to preserve space, only results from women were depicted), F1 decreased with age, especially for vowels [a] and [o], but increased slightly for vowels [i], [i]

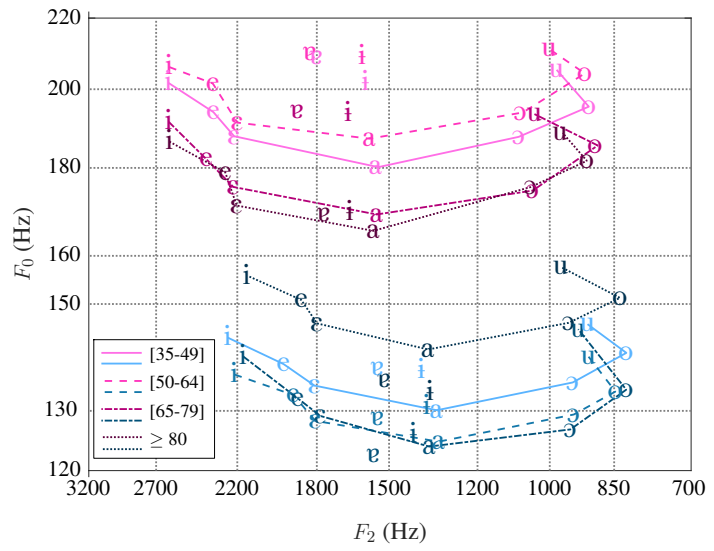

Figure 2: Mean FO as a function of vowel and age group. Top: women; bottom: men.

and [e]. Thus, for F1, ANOVA indicated that there were no significant age differences $(F(3 ; 103)=0.8 ; p=0.482)$, but suggested a significant interaction between age and vowel $(\mathrm{F}(9.1 ; 312.4)=2.4$; $\mathrm{p}=0.011)$, and between gender and vowel $(\mathrm{F}(3.0 ; 312.4)=32.5$; $\mathrm{p}<0.001)$. The analysis of variance also revealed a main effect of vowel on $\mathrm{F} 1(\mathrm{~F}(3.0 ; 312.4)=1564.9 ; \mathrm{p}<0.001)$. The main determiner of $\mathrm{F} 1$ is the phonological vowel height: the low vowel [a] has the highest F1, followed by the lower mid vowels ([0] $>[\varepsilon]>[\mathrm{e}])$, then the higher mid vowels $([\mathrm{o}]>[\mathrm{e}]>[\mathrm{i}])$, and finally the high vowels $([\mathrm{u}]>[\mathrm{i}])$ which have the lowest $\mathrm{F} 1$.

Although F2 values were slightly lower for the vowels from the $\geq 80$ speakers (cf. Table 1 ), there were no signicant differences for $\mathrm{F} 2$ between age groups $(\mathrm{F}(3 ; 101)=0.4 ; \mathrm{p}=0.774)$, indicating stability of this formant frequency across age. The analysis of variance also revealed a large main effect of vowel $(\mathrm{F}(3.2 ; 323.3)=3492.2 ; \mathrm{p}<0.001)$. The mean value of $\mathrm{F} 2$ was higher for vowel [i] followed by $[\mathrm{e}],[\varepsilon],[\mathrm{e}],[\mathrm{i}],[\mathrm{a}],[0],[\mathrm{u}]$ and [o]. A reliable interaction between vowel and gender was found $(\mathrm{F}(3.2 ; 323.3)=61.4 ; \mathrm{p}<0.001)$. Apparently, the size of the $\mathrm{F} 2$ space was larger for women than for men: the F2s of /u/ were similar for both genders, whereas /i/ values were very different.

\subsubsection{Vowel Space Area}

As illustrated in Table 1 and in Figures 3 b) c), women's VSA values $\left(492818.9 \mathrm{~Hz}^{2} \pm 135357.9\right)$ were higher than those of men $\left(310049.0 \mathrm{~Hz}^{2} \pm 73093.3\right)$. The analysis of variance revealed a large main effect of gender on VSA $(\mathrm{F}(1 ; 101)=1409.9$; $\mathrm{p}<0.001)$. For both genders, the highest mean value of VSA was observed for the age group [35-49]. VSA continuously decreased with age for men. For women VSA decreased from the age group [35-49] to [50-65], it increased over the range [65-79], and then decreased in the older age. The ANOVA showed no statistical effect of age on VSA $(F(3 ; 101)=1.1 ; p=0.339)$.

\section{Discussion}

The current study examined similarities and differences in EP vowel acoustics that male and female speakers presented in response to ageing. The findings suggest that some characteristics seem to change with age, mainly the duration and F0, and all acoustic parameters have shown to be gender dependent (except duration). Vowels duration significantly increased in both genders with ageing, which is in agreement with the literature $[19,35,3,18]$. This may be related to the slowing of the nerve 


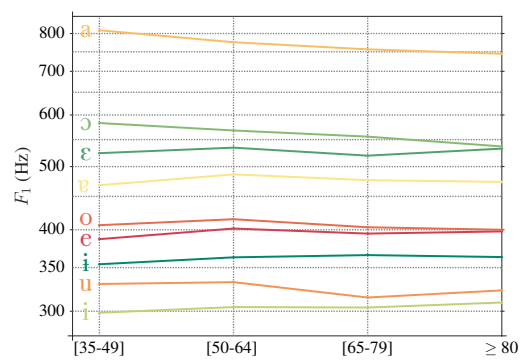

(a) Mean F1 of the EP vowels by age group

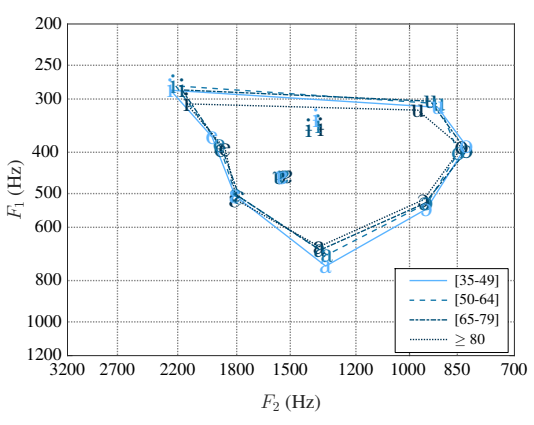

(b) Vowel Space for men

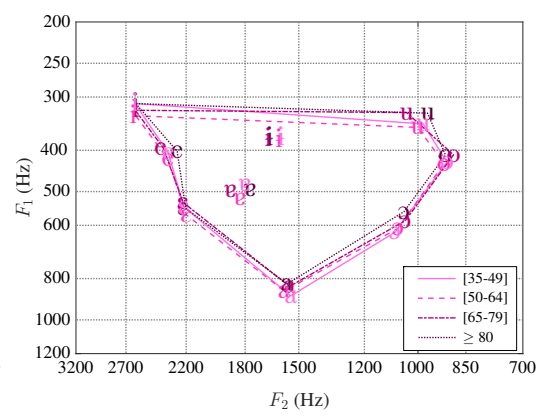

(c) Vowel Space for women

Figure 3: Age and vowel effects in first and second formant

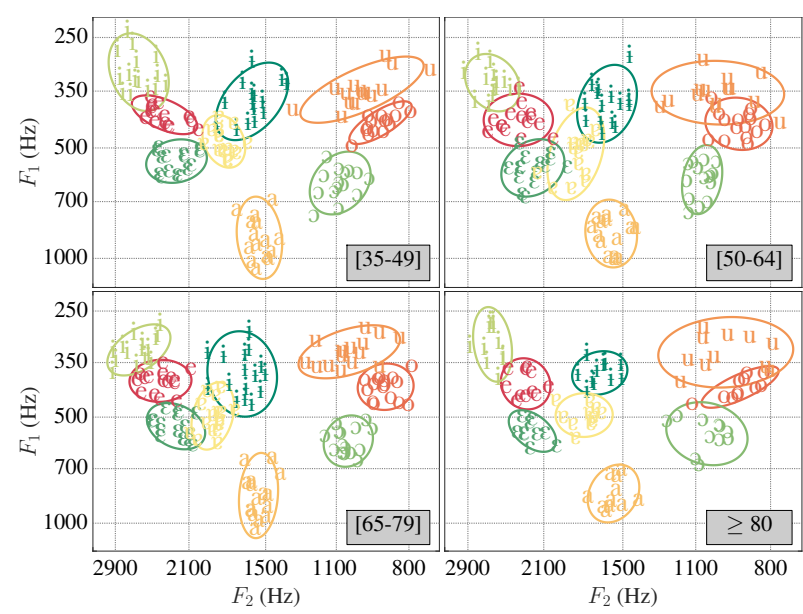

Figure 4: Mean Vowel Cluster Size for female age groups

conduction velocity and with the changes verified in the respiratory and central nervous systems [1].

F0 tends to approach between genders as age increases. The decreases of $\mathrm{F} 0$ in women is consistent with the data available in other languages [5, 6, 7, 3, 8, 9] and in EP [20, 21], and has been attributed to the endocrinological changes that occur after menopause [7, 36, 7, 1, 37]. For men there is less agreement across studies, and our results tend to confirm the trend that F0 decreases until middle age and increases again at an advanced age $[1,7,2,3,11,8]$. For EP, the few available data didn't show significant changes with age [21]. The increase of F0 in males may be associated with the muscle atrophy, or with an increase in stiffness of vocal folds tissue with ageing $[36,37,1]$.

It is clear from our results that vowel formants do not systematically decrease with age, in contrast with some previous reports $[1,14,13]$. There are vowels that presented a different pattern of formant frequencies variation with age and gender. Age related changes in F1 and F2 might be related to specific articulatory adjustments of the older speakers during speech, rather than generalized processes such as lengthening of the vocal tract $[13,5]$. Age-related changes in VSA, although not significant, show a slightly decrease, mainly for males, which supports a trend towards the centralization of vowels' space with ageing. That is vowel articulation becomes more centralized in older speakers $[15,16,26]$. And still, the smaller polygon of the older males tends to indicate that they have a worse articulation capability [26, 27].

Finally, the present study finds several general proprieties of Portuguese vowels [23], which they have in common with other languages: significant differences by gender for all vowels in F0 and formants; the high vowels have a higher F0 than low vowels, i.e. they exhibit intrinsic F0; back vowels present higher F1 than their front counterparts. The differences of F1 between front and back vowels decrease with ageing (see Figure 3 a)). So, the age group $\geq 80$ present a lower front-back distinction compared to all the other age groups.

\section{Conclusions}

This study adds to the growing body of data on the effects of age on the acoustic properties of speech, providing information on vowel acoustics from adults who speak a language different from English. In that sense, it might help to better understand crosslinguistic similarities and language-particular features of vowel ageing. Moreover, this normative data for EP vowel acoustics are important as reference for clinical assessment and treatment of different speech disorders, that are often age-related, and to provide information for speech technologies.

Several features of this research are notable. A new database was devised, containing all EP oral vowels in similar word contexts; vowels were produced by a large sample of healthy adults in four age groups; they were collected using standardized recording procedures; data segmentation was manually checked by experts; and analyses were conducted for several acoustic parameters (duration, F0, F1, F2 and VSA).

This work is the starting point for a broader life span study, involving a large number of EP speakers, from infancy to old age. The relation between the vowel acoustic and the articulatory changes with ageing should be addressed using advanced instrumental techniques, such as ultrasonography and magnetic ressonance imaging.

\section{Acknowledgements}

This research was financially supported by the project Vox Senes POCI-01-0145-FEDER-03082 (funded by FEDER, through COMPETE2020 - Programa Operacional Competitividade e Internacionalização (POCI), and by national funds (OE), through FCT/MCTES), by the grant SFRH/BD/115381/2016, and by IEETA (UID/CEC/00127/2019), and by CIDMA (UID/MAT/04106/2019). We are very grateful to the institutions for having made possible the data collection, and also to all the adults who contributed as speakers. 


\section{References}

[1] S. E. Linville, Vocal aging. Australia; San Diego: Singular Thomson Learning, 2001.

[2] H. Mautner, "A Cross-System Instrumental Voice Profile of the Aging Voice: With Considerations of Jaw Posture Effects," Ph.D. dissertation, University of Canterbury, 2011.

[3] S. Schötz, Perception, analysis and synthesis of speaker age, ser. Travaux de l'Institut de linguistique de Lund 47 . Linguistics and Phonetics, 2006.

[4] R. Vipperla, S. Renals, and J. Frankel, "Ageing voices: The effect of changes in voice parameters on ASR performance," EURASIP Journal on Audio, Speech, and Music Processing, vol. 2010, p. 5, 2010.

[5] J. T. Eichhorn, R. D. Kent, D. Austin, and H. K. Vorperian, "Effects of Aging on Vocal Fundamental Frequency and Vowel Formants in Men and Women," Journal of Voice, 2017.

[6] H. Goy, D. N. Fernandes, M. K. Pichora-Fuller, and P. van Lieshout, "Normative voice data for younger and older adults," Journal of Voice, vol. 27, no. 5, pp. 545-555, 2013.

[7] E. P. M. Ma and A. L. Love, "Electroglottographic Evaluation of Age and Gender Effects During Sustained Phonation and Connected Speech," Journal of Voice, vol. 24, no. 2, pp. 146-152, 2010.

[8] P. Torre III and J. A. Barlow, "Age-related changes in acoustic characteristics of adult speech," Journal of Communication Disorders, vol. 42, pp. 324 - 333, 2009.

[9] A. Yamauchi, H. Yokonishi, H. Imagawa, K.-I. Sakakibara, T. Nito, N. Tayama, and T. Yamasoba, "Age-and gender-related difference of vocal fold vibration and glottal configuration in normal speakers: analysis with glottal area waveform," Journal of Voice, vol. 28, no. 5, pp. 525-531, 2014.

[10] S. A. Xue and D. Deliyski, "Effects of aging on selected acoustic voice parameters: preliminary normative data and educational implications," Educational Gerontology, vol. 27, no. 2, pp. 159168, 2001.

[11] E. T. Stathopoulos, J. E. Huber, and J. E. Sussman, "Changes in acoustic characteristics of the voice across the life span: measures from individuals 4 - 93 years of age," J Speech Lang Hear Res, vol. 54, no. 4, pp. 1011-1021, 2011.

[12] L. Ramig and R. Ringel, "Effects of Physiological Aging on Selected Acoustic Characteristics of Voice," J Speech Hear Res, vol. 26, no. 1, pp. 22-30, 1983.

[13] S. A. Xue and G. J. Hao, "Changes in the Human Vocal Tract Due to Aging and the Acoustic Correlates of Speech Production: A Pilot Study," J Speech Lang Hear Res, vol. 46, no. 3, pp. 689-701, 2003.

[14] P. J. Watson and B. Munson, "A comparison of vowel acoustics between older and younger adults," in ICPhS XVI, Saarbrücken, 2007, pp. 561-564.

[15] M. Rastatter and R. Jacques, "Formant frequency structure of the aging male and female vocal tract," Folia phoniatrica, vol. 42 , no. 6, pp. 312-319, 1990.

[16] M. Rastatter, R. McGuire, J. Kalinowski, and A. Stuart, "Formant frequency characteristics of elderly speakers in contextual speech," Folia Phoniatrica et Logopaedica, vol. 49, no. 1, pp. 1-8, 1997.

[17] A. R. Fletcher, M. J. McAuliffe, K. L. Lansford, and J. M. Liss, "The relationship between speech segment duration and vowel centralization in a group of older speakers," J. Acoust. Soc. Am., vol. 138, no. 4, pp. 2132-2139, 2015.

[18] B. L. Smith, J. Wasowicz, and J. Preston, "Temporal Characteristics of the Speech of Normal Elderly Adults," J Speech Hear Res, vol. 30, no. 4, pp. 522-529, 1987.

[19] B. J. Benjamin, "Phonological performance in gerontological speech," Journal of Psycholinguistic Research, vol. 11, no. 2, pp. $159-167,1982$
[20] I. Guimarães and E. Abberton, "Fundamental frequency in speakers of Portuguese for different voice samples," Journal of voice, vol. 19, no. 4, pp. 592-606, 2005.

[21] T. Pellegrini, A. Hämäläinen, P. B. de Mareil, M. Tjalve, I. Trancoso, S. Candeias, M. S. Dias, and D. Braga, "A corpus-based study of elderly and young speakers of European Portuguese: acoustic correlates and their impact on speech recognition performance," in INTERSPEECH, 2013.

[22] L. Albuquerque, C. Oliveira, A. Teixeira, P. Sa-Couto, J. Freitas, and M. S. Dias, "Impact of age in the production of European Portuguese vowels," in INTERSPEECH, 2014.

[23] P. Escudero, P. Boersma, A. S. Rauber, and R. Bion, "A crossdialect acoustic description of vowels: Brazilian and European Portuguese," J. Acoust. Soc. Am., vol. 126, no. 3, 2009.

[24] C. Oliveira, M. M. Cunha, S. Silva, A. Teixeira, and P. Sa-Couto, "Acoustic Analysis of European Portuguese Oral Vowels Produced by Children." in Advances in Speech and Language Technologies for Iberian Languages. Springer, 2012, vol. CCIS 328, pp. 129138.

[25] M. R. D. Martins, "Análise acústica das vogais orais tónicas em Português,” Boletim de Filologia, vol. XXII, pp. 303-314, 1973.

[26] T. Arias-Vergara, J. C. Vásquez-Correa, and J. R. Orozco-Arroyave, "Parkinson's disease and aging: analysis of their effect in phonation and articulation of speech," Cognitive Computation, vol. 9, no. 6 , pp. 731-748, 2017.

[27] C. Fougeron and N. Audibert, "Testing Various Metrics for the Description of Vowel Distortion in Dysarthria," in ICPhS, 2011, pp. 687-690.

[28] C. Draxler and K. Jänsch, "SpeechRecorder," 2017.

[29] T. Kisler, U. Reichel, and F. Schiel, "Multilingual processing of speech via web services," Computer Speech \& Language, vol. 45 pp. 326-347, 2017.

[30] P. Boersma and D. Weenink, "Praat: doing phonetics by computer," 2012.

[31] J. Veloso, "Schwa in European Portuguese: The Phonological Status of [i]," in JEL'2007, Nantes, 2007, pp. 55-60.

[32] R. Smiljanic and R. C. Gilbert, "Acoustics of clear and noiseadapted speech in children, young, and older adults," J Speech Lang Hear Res, vol. 60, no. 11, pp. 3081-3096, 2017.

[33] A. T. Neel, "Vowel space characteristics and vowel identification accuracy," J Speech Lang Hear Res, vol. 51, no. 3, pp. 574-585, 2008.

[34] D. McCloy, R. Wright, and P. Souza, "Modeling intrinsic intelligibility variation: vowel-space size and structure," in Proceedings of Meetings on Acoustics 164ASA, 2014.

[35] E. Jacewicz, R. A. Fox, C. O’Neill, and J. Salmons, “Articulation rate across dialect, age, and gender," Language variation and change, vol. 21, no. 02, pp. 233-256, 2009.

[36] M. B. Higgins and J. H. Saxman, "A Comparison of Selected Phonatory Behaviors of Healthy Aged and Young Adults," J Speech Hear Res, vol. 34, no. 5, pp. 1000-1010, 1991.

[37] S. Sebastian, S. Babu, N. E. Oommen, and A. Ballraj, "Acoustic measurements of geriatric voice," Journal of Laryngology and Voice, vol. 2, no. 2, p. 81, 2012. 